\title{
Management of Open Apex in Maxillary Canine Associated with Large Periapical Cyst: A Case Report
}

\author{
Dr Aastha Aggarwal ${ }^{1}$, Dr Shashikala $\mathrm{K}^{2}$ \\ ${ }^{1}$ Post Graduate Student, ${ }^{2}$ Professor and Vice Principal Department of Conservative Dentistry and Endodontics, \\ D.A.P.M.R.V Dental College, Bangalore
}

\begin{abstract}
Aim of periapical surgery is to remove periapical pathology for complete wound healing and regeneration of bone. A large maxillary cyst was treated for 6 months by conservative approach followed by surgical therapy and cystectomy. To induce bone regeneration platelet rich fibrin was used which release growth factors after cellular interactions.
\end{abstract}

Keywords: Periapical surgery; Biodentine; Platelet rich factor; $C B C T$

\section{Introduction}

Traumatic dental injuries are usually common in anterior teeth of young patients. Such trauma is often followed by necrotic pulp which gives opportunity to bacteria from oral environment to enter the pulp chamber and root canal. ${ }^{[1]}$ This colonization of bacteria inside the tooth causes leakage of their products and toxins through the apical foramen resulting in inflammation of periapical tissue thus causing apical periodontitis. ${ }^{[2]} \mathrm{A}$ number of chemical mediators of inflammation such as cytokines IL-1a, $1 \mathrm{~b}, \mathrm{TNFa}$,prostaglandins and lipopolysaccharides seem to be related to pathogenesis of periapical lesion. ${ }^{[3]}$ Microbial attack and host response events result in apical granulation tissue that is resorption of periradicular bone and degradation of apical periodontal ligament which may turn into cyst. Epithelial lining of radicular cyst arises from epithelial cell rests of malaseez in periodontal ligament. ${ }^{[4]}$ Such lesions are usually diagnosed during routine radiographic examination or following acute pain or swelling in relation to affected tooth. ${ }^{[1]}$ Incidence of radicular cyst is highest in maxillary anterior region presumably as a result of trauma. ${ }^{[4]}$ Incidence of cyst within periapical lesion varies $\mathrm{b} / \mathrm{w} 6-55 \%$. Occurrence of periapical granulomas is $\mathrm{b} / \mathrm{w} 9.3-87 \%$ and abscess $\mathrm{b} / \mathrm{w} 28.7-70.07 \%$. ${ }^{[5]}$ This case report demonstrates successful surgical management of a large periapical lesion using Biodentine as a retrograde filling material with 12 months follow up.

\section{Case Report}

A 25 year old male patient was referred to department of cons and endo for endodontic treatment of left maxillary canine. Patient revealed history of trauma 10 years back. Intraoral clinical examination revealed an abscess on gingiva buccally in relation to 23 as well as a volumetric swelling present on palate extending from 22 to 24 [Figure 1]. Palatal swelling was soft, non-tender approx. $2.5 \times 3.5 \mathrm{~cm}$ in diameter. Pulpal necrosis was confirmed by heat test in relation to 22,23. OPG revealed radiolucent lesion around 21,22,23,24 and canine (23) with open apex [Figure 2]. Occlusal radiographs revealed well circumscribed radiolucent lesion eroding hard palate involving 21,22,23,24[FIGURE 3]. Extent of palatal bone perforation by cyst was evaluated by CBCT[FIGURE 4,5]. After confirmation of lesion with CBCT treatment was planned. After teeth isolation access cavities were prepared on 21,22,23,24. A mucous and transparent exudate drained through radicular canal of 23. Canals were irrigated by chlorhexidine $2 \%$ and sodium hypochlorite $5.25 \%$ followed by cleaning and shaping and placement of intracanal medicament of calcium hydroxide which was renewed every 15 days for 6 months. Radiographic examination at 6 months proved no trace of healing and hence periapical surgery was scheduled. 21,22,24 were obturated using cold lateral condensation technique and 23 using roll cone technique[FIGURE 6]. Under local anaesthesia palatal flap was reflected and entire cystic lesion was exposed[FIGURE 7]. Cystic lining was enucleated [FIGURE 8] and sent for histopathological examination. Root end resection of $2 \mathrm{~mm}$ for 23 was performed followed by retrograde placement of biodentine. Platelet rich factor (PRF) was prepared in accordance with the protocol developed by choukroun et al. $8 \mathrm{ml}$ intravenous blood was collected in $10 \mathrm{ml}$ sterile tube without anticoagulants and immediately centrifuged at $3000 \mathrm{rpm}$ for $10 \mathrm{mins}$. Blood centrifugation allowed formation of structured fibrin clot in the middle of the tube between red corpuscles at bottom \& acellular plasma at the top. PRF was separated from red corpuscles using a sterile tweezers \& scissors after removal from tube and transferred to sterile dappen dish. PRF was augmented into intrabony defect upto the surrounding bone level and palatal flap was repositioned and simple interrupted absorbable sutures were given. Post- operative IOPA was taken [Figure 9]. Post-operative instructions were given to the patient. Recall examination after 3,6,9 and 12-month interval was done [Figure 10,11]. 


\section{Figures}

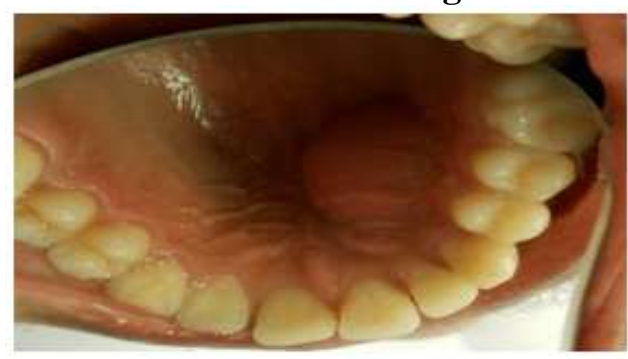

Fig. 1- Palatal swelling

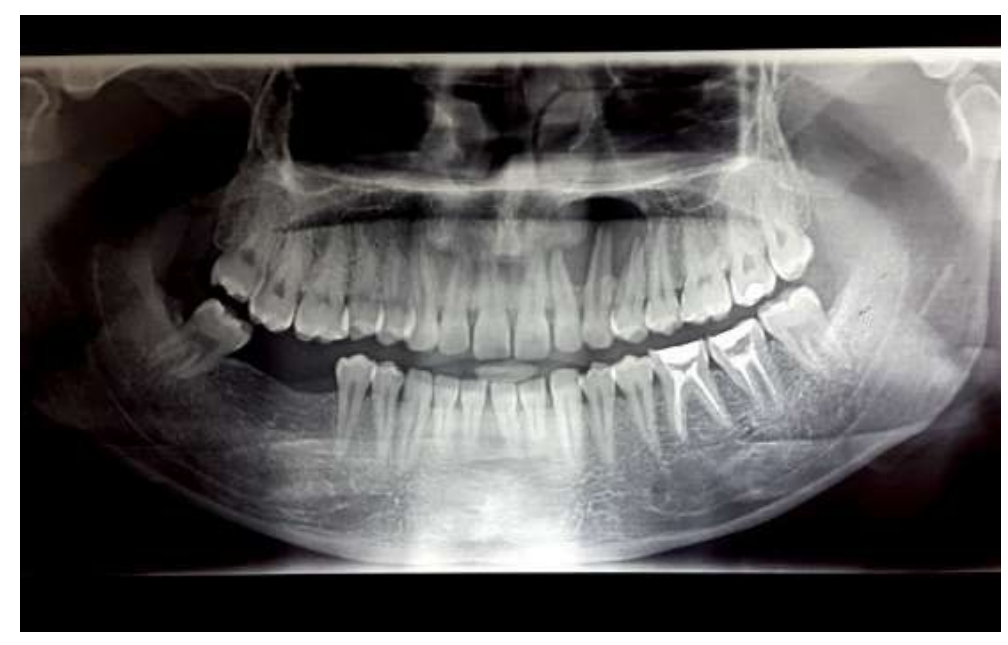

Fig. 2- Pre operative OPG showing periapical radiolucency and canine with open apex

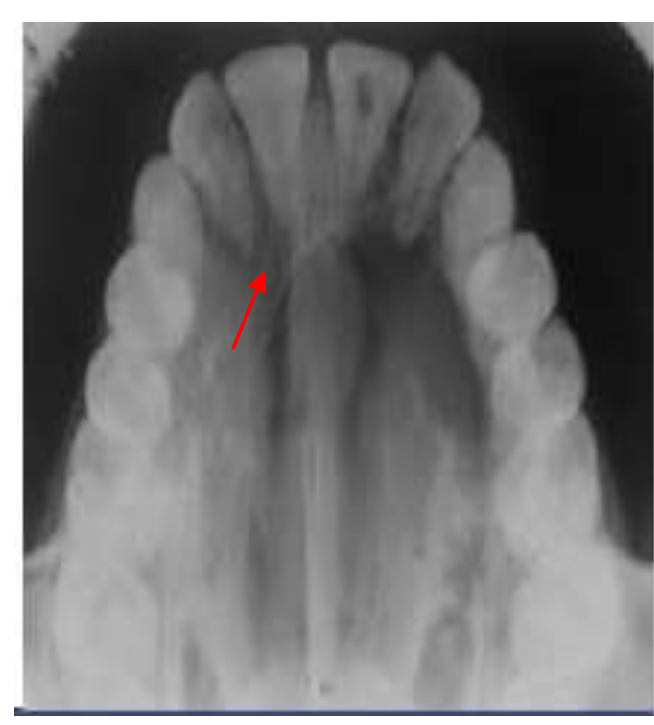

Fig. 3- Pre operative occlusal radiograph

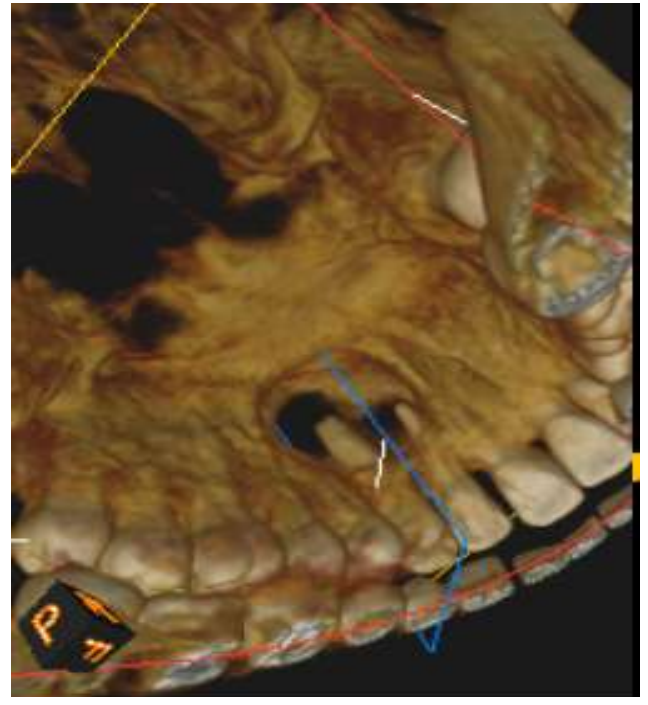

Fig. 4- CBCT showing palatal bone perforation 


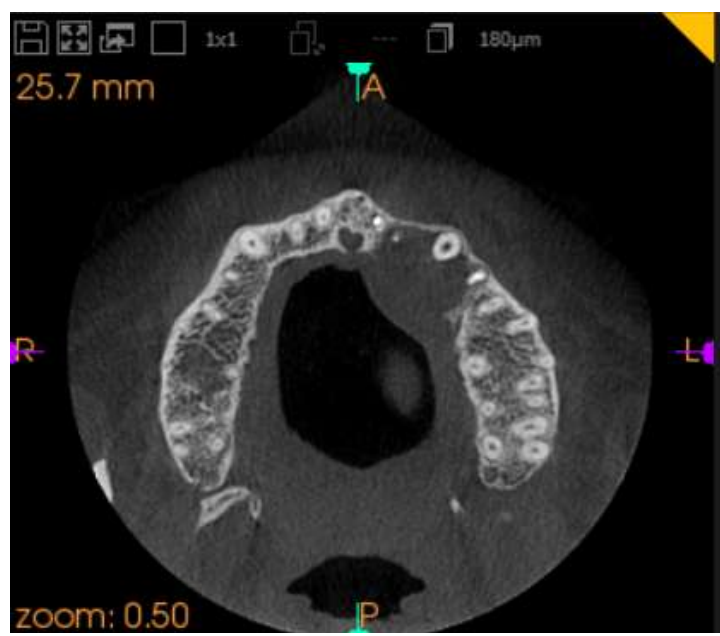

Figure 5 - CBCT showing extent of lesion

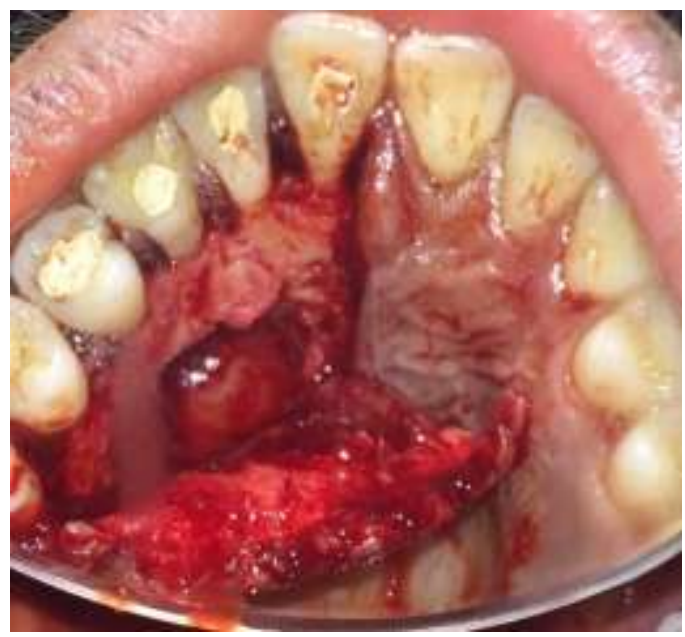

Fig. 7 - Exposure of cystic lining

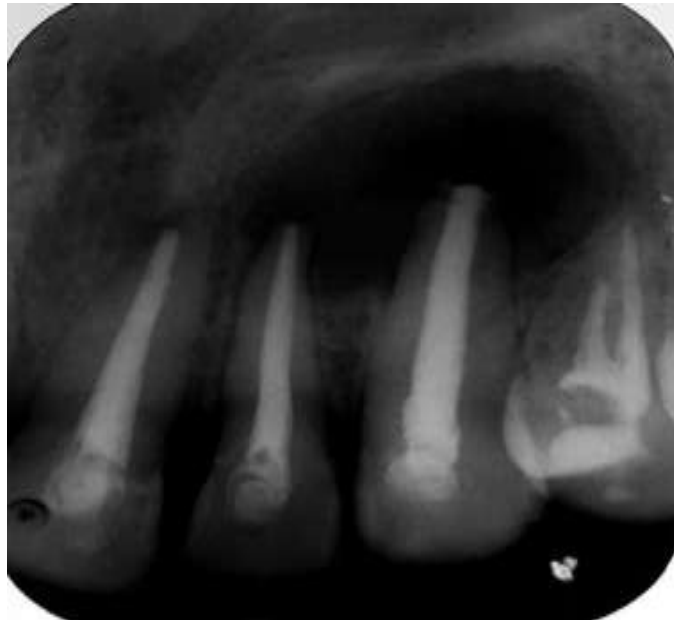

Fig. 9 - Root end resection and etrograde placement of biodentine

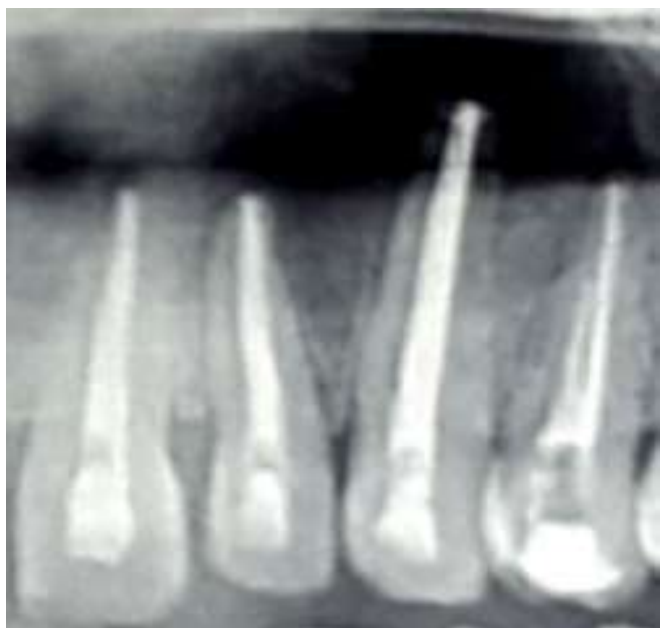

Fig. 6 - Obturation

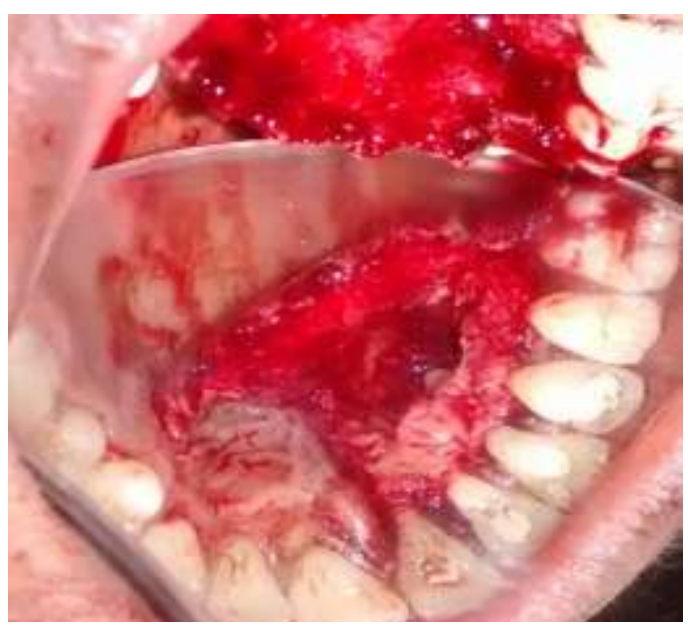

Fig. 8 -enucleation of cyst and root end resection

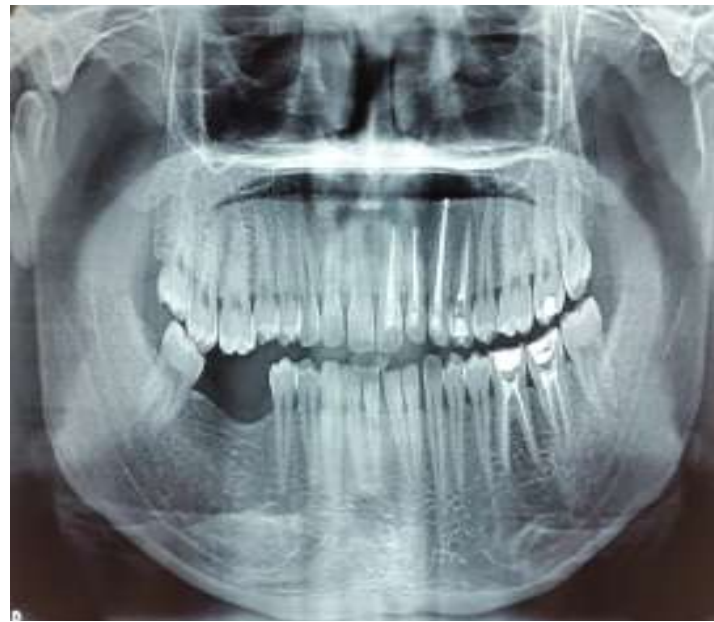

Fig. 10 - 9 month follow up showing healing of lesion 


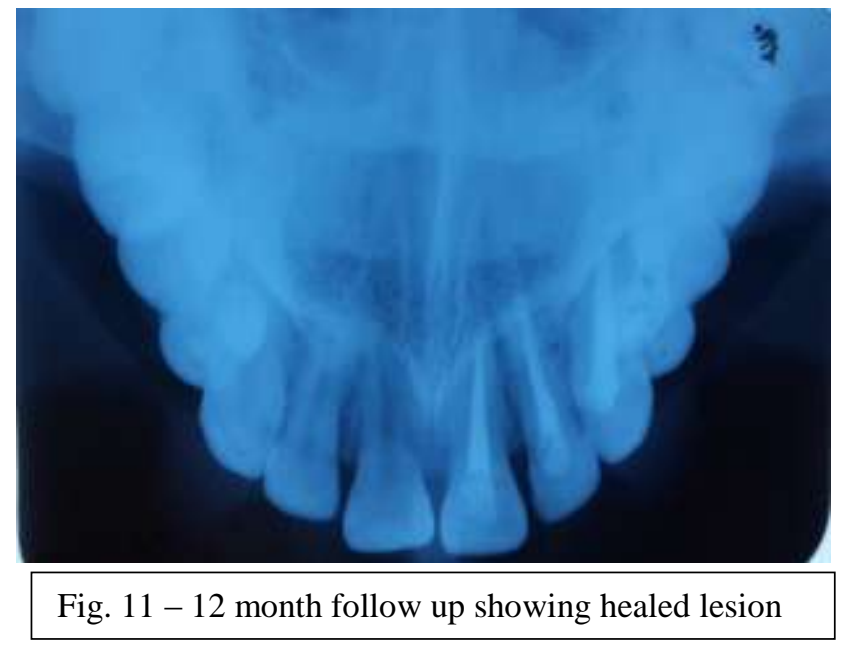

\section{Discussion}

Cyst is a pathological cavity with an epithelial carpet filled with liquid, semi liquid or gaseous content and a well-defined wall of connective tissue. Growth of a cyst is typically slow, centrifugal and infiltrative. ${ }^{[1]}$ There are two distinct categories of radicular cyst namely those containing cavities which is completely enclosed by epithelial lining, true cyst and those containing epithelial lined cavities that are open to the root canals (Simon 1980, Nail et al 1996). Later was described as a "bay cyst" (Simon 1980) and now designated as periapical pocket cyst (Nair et al 1996). ${ }^{[4]}$

Root apex is of interest to endodontist. Development of root begins after enamel and dentin formation has reached future CEJ. At the time of tooth eruption only $2 / 3$ rd of root is formed. Due to trauma/ caries exposure pulp may undergo necrosis, dentin formation ceases and root growth is arrested. Thus the resulting immature root will have an open apex also known as blunderbuss canal. ${ }^{[6]}$

Goal of periradicular surgery is to gain access to the affected area, evaluate root circumference and its anatomy and place a biocompatible seal in form of root end filling that stimulates regeneration of periapical tissue. The periapical modality for managing failure of conventional orthograde endodontic treatment in case of a large non healing periapical lesion is apical surgery with success rate $86-92 \% .{ }^{[2]}$

Kolkate and Pawar conducted a study that compared microleakage of GIC, MTA and Biodentine when used as a retrograde filling material and concluded that Biodentine exhibited the least microleakage. ${ }^{[2]}$ Biodentine powder contains tricalcium silicate, calcium carbonate and dicalcium silicate, zirconium oxide and liquid consist of calcium carbonate in aqueous solution with an admixture of polycarbonate. It possess better physical and biological properties in comparison to other tricalcium silicate based cements.

Major advantage of CBCT is its three dimensional geometric accuracy compared to the conventional radiographs. Sagittal, coronal and axial view of CBCT images eliminate the superimposition of anatomical structures ${ }^{[7]}$ Bone regeneration after periapical surgery is influenced by four critical factors - primary wound closure, angiogenesis as a blood supply and source of undifferentiated mesenchymal cells, space maintenance and stability of wound (PASS Principle).${ }^{[8]}$ PRF helps to obtain fibrin membranes enriched with platelets and growth factors. PRF releases autologous growth factors gradually for atleast 1 week and upto 28 days. ${ }^{[5]}$ PRF attempts to concentrate platelets and release cytokines in a fibrin clot. ${ }^{[8]}$

\section{CONCLUSION}

CBCT has major role in diagnostic field in endodontics. In the present study its application helps to access the site and extent of large periapical cyst and its proximity to anatomical structures enables the clinician in better management of periradicular surgery. Open apex could be successfully managed with the use of biodentine in the study.

\section{REFERENCES}

[1]. Torres-Lagares, Daniel, et al. "Treatment of a large maxillary cyst with marsupialization, decompression, surgical endodontic therapy and enucleation." J Can Dent Assoc 77.77 (2011): b87.

[2]. Pawar, Ajinkya M., Sharad R. Kokate, and Reema A. Shah. "Management of a large periapical lesion using Biodentine TM as retrograde restoration with eighteen months evident follow up." Journal of Conservative Dentistry 16.6 (2013): 573.

[3]. Vier, Fabiana Vieira, and José Antônio Poli de Figueiredo. "Prevalence of different periapical lesions associated with human teeth and their correlation with the presence and extension of apical external root resorption." (2002).

[4]. Çalışkan, M. K. "Prognosis of large cyst-like periapical lesions following nonsurgical root canal treatment: a clinical review." International Endodontic Journal 37.6 (2004): 408-416. 
[5]. Jayalakshmi, K. B., et al. "Platelet-rich fibrin with $\beta$-tricalcium phosphate—a noval approach for bone augmentation in chronic periapical lesion: a case report." Case reports in dentistry 2012 (2012).

[6]. Salgar, AVINASH R., MANOJ G. Chandak, and N. U. Manwar. "Blunder buss canal: A challenge for endodontist." Endodontology 23.1 (2011): 77-81.

[7]. Patel, S., et al. "The potential applications of cone beam computed tomography in the management of endodontic problems." International endodontic journal 40.10 (2007): 818-830.

[8]. Meshram, Vikas Sukhadeo, et al. "The autologous platelet rich fibrin: A novel approach in osseous regeneration after cystic enucleation: A pilot study."Indian Journal of Dental Research 26.6 (2015): 560. 